\title{
Toward Dynamic Adoption for a User's Situation Information in a Context-Aware Workflow System ${ }^{\star}$
}

\author{
Yongyun Cho, Kyoungho Shin, Jongsun Choi, and Jaeyoung Choi \\ School of Computing, Soongsil University, \\ 1-1 Sangdo-dong, Dongjak-gu, Seoul 156-743, Korea \\ \{yycho, delio, jschoi\}@ss.ssu.ac.kr, choi@comp.ssu.ac.kr
}

\begin{abstract}
Recently, there are many studies to adopt the workflow model, which has been successively applied to traditional computing environments, into ubiquitous computing environments for context-aware and autonomous services. A service in the ubiquitous computing environments must be executed according to a user's situation information, which is generated dynamically from sensors. However, such existing workflow systems as FollowMe and uFlow to support context-aware services through workflow models, can't immediately adopt changes of a user's situation into a already on-going service workflow. In this paper, we propose a context-aware workflow system, for ubiquitous computing environments, which can apply changes of user's service demand or situation information into an on-going workflow without breaking its operation. To do this, the proposed system represents contexts described in a workflow as a RDF-based DItree (Document Instance tree). The system uses the tree information to recognize a exact position to be changed in the on-going workflow for user's situation changes, and to reconstruct only the position under the influence of the changes in the DItree. Therefore, the suggested system can quickly and efficiently apply a change of user's new situation into a on-going workflow without a lot loss of the time and the space, and can offer a context-aware service continuously, according to a new workflow.
\end{abstract}

\section{Introduction}

A workflow model for business services in traditional distributed computing environments can be applied as a service model to connect services with others related in ubiquitous computing environments and express service flows [1]. Compared with traditional distributed computing environments, workflow services in ubiquitous computing environments must decide a service transition according to the user's situation information that is dynamically generated from various sensors in ubiquitous environments 4. For that, a workflow system in ubiquitous environments must consider the user's situation information in service

\footnotetext{
* This work was supported by Korea Research Foundation Grant (KRF-2006-005J03803).
} 
executions of workflows. Workflow systems such as FollowMe and uFlow can supply context-aware services through workflows, which express user's situation services as service's execution conditions. Usually in the ubiquitous computing environments, the information dynamically occurs, and frequently changes initial conditions to execute a service. However, the existing workflow systems cannot apply the dynamically occurred changes into an on-going service workflow. Therefore, when changes of a user's service request or his situation information happen dynamically, we need a method that can re-apply the changes in a scenario and supply a context-aware service correspondent with the changes.

In this paper, we propose a context-aware workflow service system that uses contexts in a workflow service scenario as conditions of service execution, and dynamically derives service transition according to a user's situation information generated from real environments. To do this, the suggested system represents contexts described in the scenario as rule-based context subtrees. When a change of a user's situation information happens, the suggested system can dynamically reconstructs a workflow by modifying only the subtrees under the effect of ethe change. It means that the suggested system does not obstruct a flow of an earlier on-going context-aware service. Therefore, the suggested system uses the modified sub-tree's node information in comparison with user's situation information, and can support context-aware service continuously without stopping a on-going workflow.

\section{Related Work}

\subsection{Workflow Languages for Context-Aware Services}

From the studies to adopt workflow models to ubiquitous computing environments, we know that a workflow in a ubiquitous computing environment should consider not only result values but also context information as transition constraint for service execution. Unlike such workflow languages as BPEL4WS 5, WSFL [6], and XLANG [7], which do not consider a context information as transition conditions of services, uWDL 3. can describe context information as transition conditions of services through the $<$ context $>$ element consisting of the knowledge-based triplet - subject, verb, and object. uWDL reflects the advantages of current workflow languages such as BPEL4WS, WSFL, and XLANG, and also contains rule-based expressions to interface with the DAML+OIL 8, ontology language.

\subsection{Workflow Systems for Context-Aware Services}

WorkSco [10] is an situation-adaptable workflow system that can support service demands generated dynamically in a business process. It is based on a micro workflow model, a dynamic evolution and an open-point adaptation techniques to dynamically handle user's requests, which may be generated in various business domains. However, even though WorkSco considers dynamic handling for user's requests in a workflow system, it does not yet give an explicit method 
to do that in ubiquitous computing environments. FollowMe 11 is an OSGi framework that unifies a workflow-based application model and a context model based on ontology. FollowMe uses a scenario-based workflow model to handle user's service demands from various domains. However, it does not support a method to handle user's service demands during service processing. uFlow 3 is a ubiquitous workflow framework to support a context-aware service based on a uWDL workflow scenario. Because uFlow is also based a workflow scenario like FollowMe, it does not yet consider a method to handle the changes of user's demands or user's situation information, such as user's position or user's doing, which can be dynamically generated during service processing.

\section{A context-Aware Workflow Service System}

\subsection{A System Architecture}

Figure 1 shows a architecture of a suggested context-aware workflow system, which is aware of dynamic changes of user's situation information in ubiquitous computing environments.

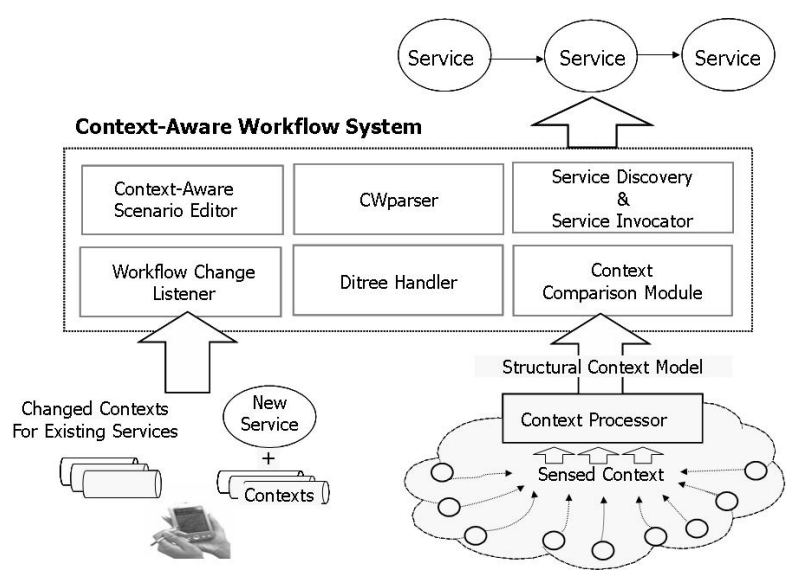

Fig. 1. An architecture of a suggested context-aware workflow system

As shown in Figure 1, a suggested system supports context-aware workflow services using a uWDL document. After a service developer or an end-user writes an uWDL workflow service scenario, the scenario is transmitted to the CWparser in Figure 1. The CWparser (Context Workflow scenario parser) represents contexts described in an uWDL scenario as RDF-based context subtrees through parsing. The CWparser constructs the RDF-based context subtree by using the structural context model [3]. And, the suggested system uses the model to objectify contexts, which is actually sensed from environments as the entities. The service discovery module searches a service appropriate to objectified contexts from an available service lists, and the service invocation module invokes the service. 


\subsection{A Context Comparison for Contex-Aware Workflow Services}

The suggested system uses uWDL as a workflow language to compose a workflow scenario for context-aware workflow service. In Figure 1, the context comparison module compares contexts described in a uWDL workflow as transition conditions for a service in a context subtree with contexts objectified as entities through the structural context model for contexts sensed from ubiquitous environments. In the comparison, the suggested system drives an execution process of the service only if the context comparison module finds objectified contexts suitable as transition conditions of a service. Figure 2 shows a context comparison algorithm.

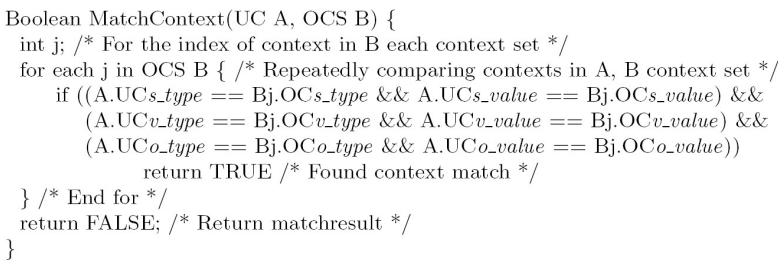

Fig. 2. An algorithm for comparing UC A with OCS B

In Figure 2, OC means a context objectified with the structural context model, and it consists of $\mathrm{OC} s, \mathrm{OC} v$, and $\mathrm{OC} o$, which mean subject, verb, and object entities, respectively. UC means a context described in a uWDL scenario. An OC consists of (OCs_type, OCs_value), (OC $v_{-}$type, OC $v_{-}$-value), (OCo_type, OCo_value), and an UC consists of (UCs_type, UCs_value) (UC $v_{-}$type, UC $v_{-}$value), (UC $s_{-}$type, $\mathrm{UC} o_{-}$value). $\mathrm{UC} s, \mathrm{UC} v$, and $\mathrm{UC} o$ mean subject, verb, object entities, respectively. A context consists of a pair of type and value. Also, OCS and UCS that mean each set of $\mathrm{OC}$ and $\mathrm{UC}$ can be defined as $\mathrm{OCS}=(\mathrm{OC} 1, \mathrm{OC} 2, \mathrm{OC} 3, \ldots, \mathrm{OC} i)$ and $\mathrm{UCS}$ $=(\mathrm{UC} 1, \mathrm{UC} 2, \mathrm{UC} 3, \ldots, \mathrm{UC} i)$.

\subsection{A Dynamic Adoption for Changes of User's Demands or Contexts}

In ubiquitous environments, a user can meet the kaleidoscope of situations, and will want a new context-aware service for the changes. However, existing contextaware workflow systems, which are almost based on a context-aware workflow scenario including contexts as transition conditions of service, cannot adopt the changes of situations into already on-going workflows. The change may be a new service demand with new contexts as transition conditions for execution of the service. And, it may be modifying of contexts, which may be used as transition conditions for a service by an on-going workflow. If a user through hand-held equipments such as PDA, or PCS raises a change, the workflow change listener instantly catches the change and throws it to the DItree handler. Then, 
the DItree handler finds parts influenced in a DItree of an on-going workflow scenario by the change and modifies only the subtrees around the parts. Figure 3 shows changes in a DItee for a sample uWDL workflow, after a user makes a new service demand including contexts as execution conditions of the service.

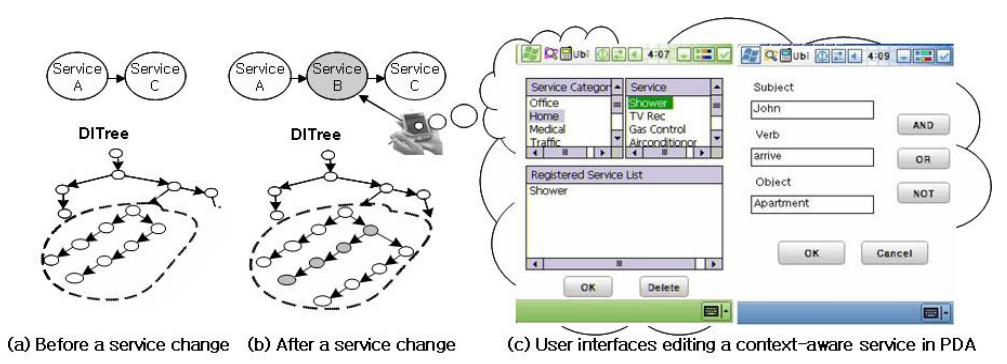

Fig. 3. Changes in a DItree when a user makes a new service and edit windows in PDA

In Figure 3(a), the suggested system will individually and automatically processes the service A and $\mathrm{C}$ according to contexts described as their execution conditions in a uWDL workflow. The dotted area in (a)'s DItree expresses RDF-based subtrees for the service A and C. Figure 3(b) represents the partial change of the DItree, when a user makes a new service, which may has to be between the service A and C orderly. A user needs interfaces to input a new service or modify a existing service through a his hand-held device. Figure 3(c) shows the workflow edit window that the suggested system offers. The workflow edit window consists of a service select window, which includes a usable service list and a registered service window, and a pop-up window to input RDF-based context information. For modifying contexts of a existing service, a user selects the service from the registered service lists in Figure 3(c). Then, he just modifies contexts through the RDF-based input fields. Now, to make a new service, a user selects a service, which he wants, from the service category window and the service list window. In the context information window of Figure 3(c), 'AND', 'OR', and 'NOT' buttons are for a composite context. Then, he has to input contexts, which are needed to execute the selected service. Figure 4 shows a demand process algorithm to adopt the changes into an on-going workflow and to do the dynamic reconstruction.

In Figure 3, because the service $\mathrm{A}$ and $\mathrm{C}$ are affected by a new service including its contexts as execution conditions, the DItree handler will re-constructs only the subtrees of the dotted area by using the demand process algorithm. The DItree's reconstruction happens partially and incrementally only in the part [12, which is influenced by workflow's changes. Therefore, the suggested system can quickly and efficiently make a new DItree including new demands or changes, with re-using the remaining parts of the DItree. 


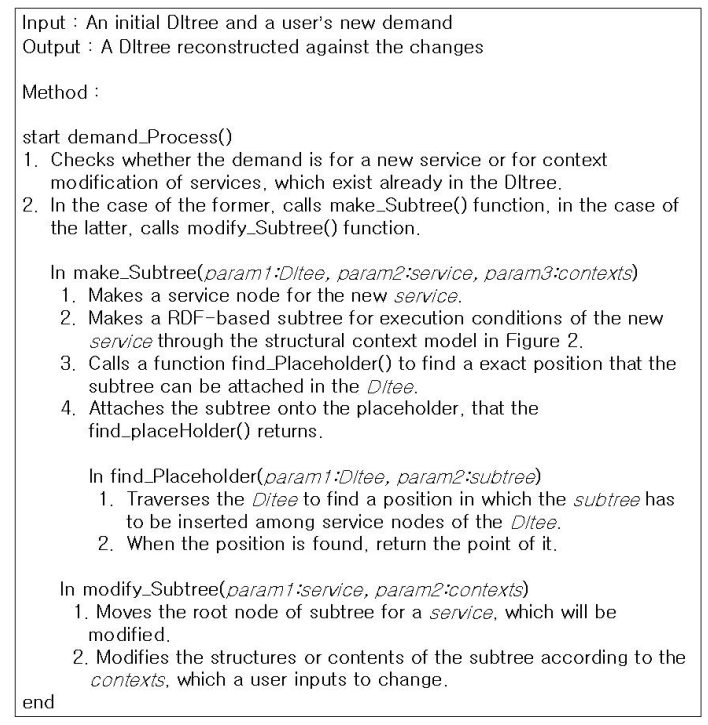

Fig. 4. An demand process algorithm to adopt changes of contexts to a DItree

\section{Experiments and Results}

For an experiment with the suggested system, we develop a workflow scenario for smart home services in ubiquitous environments, and show how the suggested system can efficiently handle service demands generated dynamically from a user, and continuously support a context-aware workflow service. The scenario was developed in a uWDL editor 3. The example scenario is as follows: John has a plan to go back his home at 10:00 PM, take a warm bath, and then watch a recorded TV program, which he wants to see after a bath. When John arrives in his apartment, an RFID sensor above the apartment door transmits John's basic context information (such as name and ID number) to the smart home server. If the conditions, such as user location, situation, and current time, are satisfied with contexts described in the workflow service scenario, then the server will prepare warm water. When he sits on the sofa in the living room after he finishes his bath, the service engine will turn on the power of the TV in the living room and play the TV program that was recorded earlier.

Now, let's again suppose that, as John is driving to his home, he needs a new service, which is a meeting preparation service with Tom at his home. The new service is to download files for meeting from John's PC in his office. That is a migration service about job environments.

If John arrives in front of his home's door with Tome, the sensed context OCs are not only for John but also for Tom. For example, the OCs may be John and Tom's locations, IDs, and Times. If John gave a priority to the meeting service during coming back to his home, the reserved bath service will be postponed after the meeting service. That is, the sensed context OCs, which are suitable 


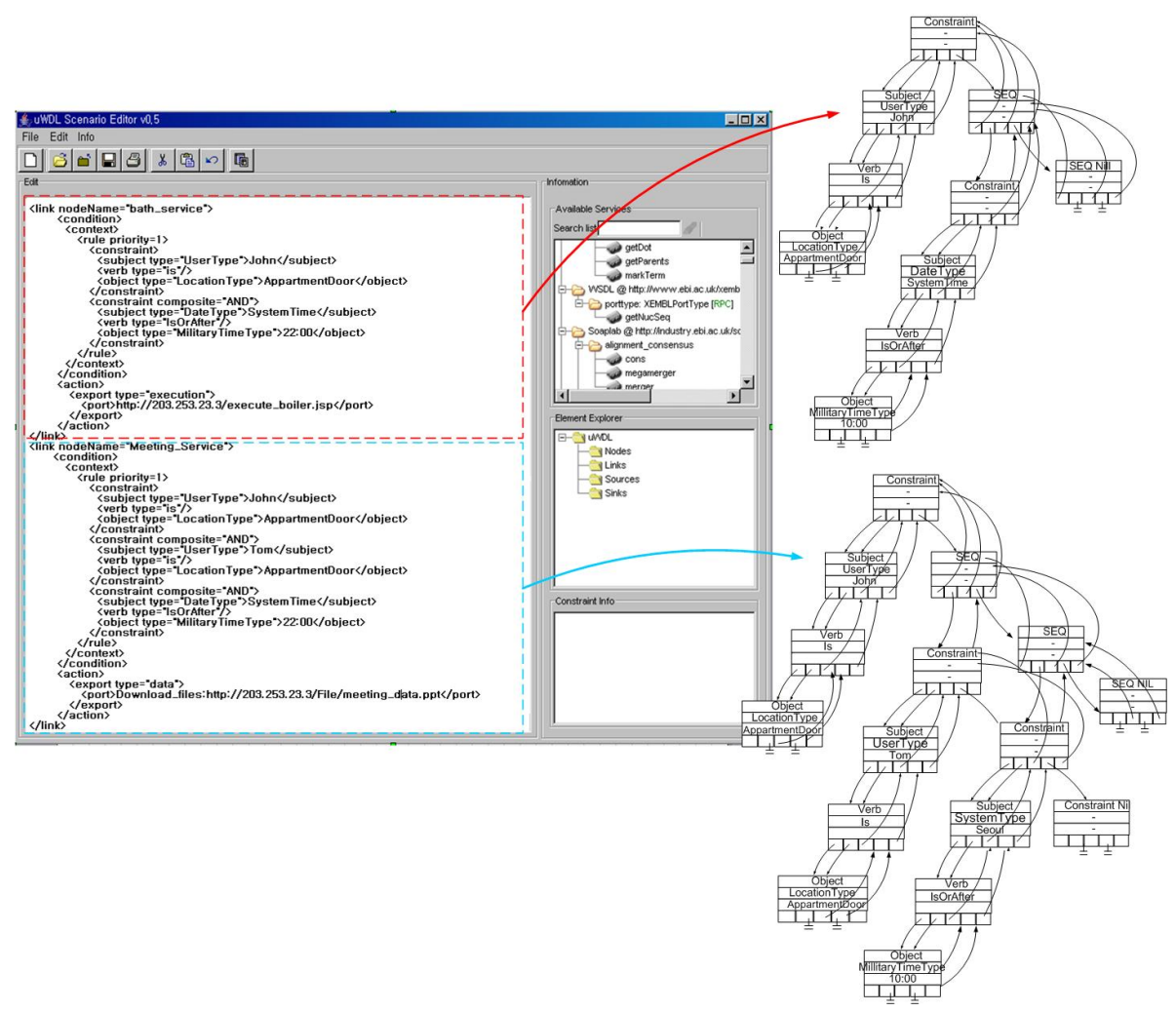

Fig. 5. The changes of the workflow scenario and its DItree

for both the bath service and the meeting service, will be applicable to the new service, not the bath service due to the priority. Figure 5 shows changes of the workflow scenario and its DItree after the DItree handler did dynamically adopt the new service to the initial workflow.

The bath service will be re-operated after Tom finishes the meeting with John, if John does not retract the bath service itself. In that time, the contexts as execution conditions of the bath service will be Tom's location and the meeting's situation. For example, if Tom locates out of John's house door and a value of the meeting's situation is over, the bath service will re-operate. After that, the suggested system executes remaining services in the workflow scenario according to contexts transmitted from the context processor.

\section{Conclusion}

In this paper we propose a context-aware workflow system dynamically to support user's service demands, by adopting changes of services or contexts into an initial workflow without interrupting the workflow. Through experiments, we 
showed that the suggested system represented contexts described in the workflow scenario as RDF-based subtrees and a process of reconstructing a DItree. The proposed system uses an demand process algorithm to support context-aware services without interrupting through recognizing exactly the place holder that have to be changed in a workflow scenario and reconstructing only the part under the influence of the changes. Through an experiment with an example workflow scenario, we showed how the suggested system can reconstruct DItree for a user's new service demand. With the suggested system, a user can easily and efficiently apply his new service demands into a scenario document regardless of the time and the space. Therefore he can be served a continuous context-aware service according to a new workflow scenario adopted with the new service demands.

\section{References}

1. Workflow Management Coalition: The Workflow Handbook 2002, Future Strategies Inc. and Lighthouse Point, FL, USA., (2002)

2. Anind k. Dey: Understanding and Using Context, Personal and Ubiquitous Computing, Vol 5, Issue 1, pp.69-78 (2001)

3. Joohyun Han, Yongyun Cho, Jaeyoung Choi: Context-Aware Workflow Language based on Web Services for Ubiquitous Computing, ICCSA 2005, LNCS 3481, pp. 1008-1017, (2005)

4. Anand Ranganathan, Scott McFaddin: Using Workflows to Coordinate Web Services in Pervasive Computing Environments, Proceedings of the IEEE International Conference on Web Services, ICWS'04, pp. 189-197, (2004)

5. Tony Andrews, Francisco Curbera, Yaron Goland: Business Process Execution Language for Web Services, BEA Systems, Microsoft Corp., IBM Corp., Version 1.1 (2003)

6. Frank Leymann: Web Services Flow Language (WSFL 1.0). IBM (2001)

7. Satish Thatte: XLANG Web Services for Business Process Design, Microsoft Corp. (2001)

8. R. Scott Cost, Tim Finin: ITtalks: A Case Study in the Semantic Web and DAML+OIL, University of Maryland, Baltimore County, IEEE (2002) 1094-7167

9. W3C: RDF/XML Syntax Specification, W3C Recommendation (2004)

10. Pedro Vieira, Antonio Rito-Silva: Adaptive Workflow Management in WorkSCo, 16th International Workshop on Database and Expert Systems Applications (DEXA'05), pp. 640-645, 2005.

11. Jun Li, Yingyi Bu, Shaxun Chen, Xianping Tao, Jian Lu: FollowMe: On Research of Pluggable Infrastructure for Context-Awareness, 20th International Conference on Advanced Information Networking and Applications(AINA'06), Volume 1, pp. 199-204, 2006

12. C. Ghezzi and D. Mandrioli: Incremental Parsing, ACM Transactions on Programming Languages and Systems, 1(1):58-70, 1979. 